\title{
Sugerencias para mejorar la regulación chilena de manipulación de vertebrados terrestres en poblaciones naturales en el contexto de investigaciones científicas
}

\section{Suggestions to improve the Chilean regulation of manipulation of terrestrial vertebrates in natural populations in the context of scientific research}

\author{
Guillermo D’Elía ${ }^{1 *}$, Fabián Jaksic ${ }^{2,3}$, Leonardo D. Bacigalupe ${ }^{1}$, Francisco Bozinovic ${ }^{2,3}$, Jhoann L. \\ Canto $^{4}$, Claudio Correa ${ }^{5}$, Francisco E. Fontúrbel ${ }^{6}$, Fulgencio Lisón ${ }^{7}$, Marco A. Méndez ${ }^{8}$, Roberto \\ Nespolo ${ }^{1}$, Juan C. Opazo ${ }^{1}$, R. Eduardo Palma ${ }^{2}$, Jaime R. Rau ${ }^{9}$, Sara M. Rodríguez ${ }^{10}$, Enrique \\ Rodríguez-Serrano ${ }^{5}$, Pablo Sabat ${ }^{3,8}$, Rodrigo A. Vásquez ${ }^{8}$ \& Pedro Victoriano ${ }^{5}$. \\ ${ }^{1}$ Instituto de Ciencias Ambientales y Evolutivas, Universidad Austral de Chile, campus Isla Teja s/n, Valdivia, Chile. \\ 2Departamento de Ecología, Pontificia Universidad Católica de Chile, Alameda 340, Santiago, Chile. \\ ${ }^{3}$ Center of Applied Ecology \& Sustainability (CAPES), Pontificia Universidad Católica de Chile, Alameda 340, Santiago, Chile. \\ ${ }^{4}$ Área Zoología de Vertebrados, Museo Nacional de Historia Natural, Parque Interior Quinta Normal Santiago s/n, Casilla 787, \\ Santiago, Chile. \\ ${ }^{5}$ Departamento de Zoología, Universidad de Concepción, Casilla 160-C, Concepción, Chile. \\ ${ }^{6}$ Instituto de Biología, Pontificia Universidad Católica de Valparaíso, Av. Universidad 330, Valparaíso, Chile. \\ ${ }^{7}$ Departamento de Ciencias Forestales y Medioambiente, Universidad de La Frontera, Francisco Salazar 1145, Temuco, Chile. \\ ${ }^{8}$ Departamento de Ciencias Ecológicas, Universidad de Chile, Las Palmeras 3425, Ñuñoa, Santiago, Chile. \\ 9Departamento de Ciencias Biológicas y Biodiversidad, Universidad de Los Lagos, Casilla 933, campus Osorno, Osorno, \\ Chile. \\ ${ }^{10}$ Instituto de Ciencias Marinas y Limnológicas, Universidad Austral de Chile, campus Isla Teja s/n, Valdivia, Chile. \\ *guille.delia@gmail.com
}

\begin{abstract}
RESUMEN
La manipulación con fines científicos de vertebrados terrestres en poblaciones naturales chilenas se debe realizar previa autorización del SAG y de Comités de Bioética institucionales. Obtener dichas autorizaciones es cada vez más complejo; los trámites no se adecúan al quehacer científico ni al conocimiento sobre el efecto de la manipulación en las poblaciones naturales. El objetivo de este comentario es contribuir a una discusión en pos del establecimiento de una normativa de manipulación científica de vertebrados terrestres ajustada a la realidad biológica y que no entorpezca la investigación científica.
\end{abstract}

Palabras clave: colecta científica, Comité de Bioética, conservación biológica, SAG.

\begin{abstract}
In Chile, the manipulation for scientific purposes of terrestrial vertebrates from natural populations is conducted previous authorization of the Agricultural and Livestock Service and various Bioethics Committees. Obtaining such authorizations is becoming increasingly complex. The procedures do not fit the reality of scientific work, and they seem to be based on unjustified assessments of the effects of animal handling on natural populations. The aim of this commentary is to initiate a discussion in order to establish a norm of scientific manipulation of terrestrial vertebrates adjusted to biological reality and that does not interfere with scientific research.
\end{abstract}

KEYWORDS: bioethics, biological conservation, SAG, scientific collection. 


\section{INTRODUCCIÓN}

El conocimiento científico se genera a partir del análisis de datos a la luz de antecedentes previos. Cuando se trata de conocimiento sobre aspectos de la ecología y evolución de animales vivientes, la obtención de estos datos se realiza en terreno, laboratorio o en colecciones museológicas. A su vez, la toma de datos implica, en alguna instancia directa o indirectamente, la manipulación de animales en poblaciones naturales. Dependiendo del tipo de investigación a desarrollar, la manipulación puede ser temporalmente corta y no tener mayores consecuencias para los animales (e.g., éstos se capturan y luego de un corto período, en que se determinan a nivel específico y se registran variables individuales, son liberados en el mismo lugar de captura), acarrear consecuencias menores (e.g., se toman biopsias o se instalan radiotransmisores), o bien tener efectos extremos cuando los animales son definitivamente removidos de las poblaciones naturales (ya sea para trasladarlos a condiciones experimentales controladas, o para ser sacrificados con el objetivo de obtener distintos tipos de muestras - si el trabajo está bien implementado, estos especímenes terminarán siendo ingresados en colecciones científicas donde podrán ser utilizados en futuros estudios).

En Chile, la manipulación con fines científicos de vertebrados terrestres (i.e., anfibios, reptiles, aves y mamíferos) se debe realizar previa autorización del Servicio Agrícola y Ganadero (SAG), entidad estatal dependiente del Ministerio de Agricultura. Junto a lo anterior, en los últimos años diversos Comités de Bioética de las instituciones que patrocinan o albergan tales investigaciones han cobrado protagonismo al momento de solicitar autorizaciones de manipulación de animales con fines científicos. Específicamente, las solicitudes realizadas al SAG deben contar con la aprobación del Comité de Bioética institucional correspondiente.

\section{EL PROBLEMA}

Estos trámites presentan, en líneas generales, dos problemas mayores: son engorrosos y con frecuencia las solicitudes terminan siendo aprobadas con restricciones respecto a las propuestas originales, comprometiendo en algunos casos la viabilidad de las investigaciones. Estimamos que esto es causado en primera instancia porque el trámite de solicitud no está diseñado para dar cuenta de ciertos aspectos de la naturaleza y complejidad de la investigación científica realizada en terreno. En segunda instancia, varios de los cuestionamientos al plan de trabajo propuesto, que redunda muchas veces en restricciones a la propuesta original, parecen estar basados en juicios que no se condicen con la evidencia científica (i.e., el entendimiento que tenemos de la naturaleza). Por ejemplo, cuando se plantea explorar algunas de las múltiples zonas de Chile en que no se sabe qué especies de vertebrados se encuentran, no es posible indicar a priori, como se requiere en los formularios de solicitud de colecta científica, las especies que se van a manipular y los lugares exactos donde se van a manipular. Es más, aún en zonas que se consideran bien conocidas existe la posibilidad de descubrir especies no registradas previamente. Prueba de esto es el hecho que desde 2013 a la fecha, se han descrito seis especies de mamíferos nuevas para la ciencia en base a especímenes colectados en Chile (Spotorno et al. 2013; D'Elía et al. 2015, 2016a; Teta y D'Elía 2016; Palma y Rodríguez-Serrano 2018). De igual forma, de 2014 a la fecha, otras cuatro especies de mamíferos continentales, previamente conocidas de países vecinos, han sido registradas por primera vez para Chile (Ossa et al. 2015, 2018; Valladares et al. 2015; D’Elía et al. 2016b). El escenario es similar para otros grupos de vertebrados, notablemente los reptiles (e.g., Demangel et al. 2015; Troncoso-Palacios et al. 2018). Estos hallazgos, sumados a múltiples refinamientos en las distribuciones conocidas de distintas especies, indican que cuando el foco del trabajo es caracterizar la composición faunística de un lugar, no siempre (en teoría, nunca) se puede entregar por adelantado un listado de las especies a manipular dado que muchas veces no se sabe qué especies habitan determinada área. De esto se desprende que los formularios del SAG no están diseñados para dar cuenta de parte de la actividad científica, ni tampoco del conocimiento científico actual (e.g., existen áreas geográficas mal relevadas y la diversidad real de muchos grupos de vertebrados es pobremente entendida).

En ciertas instancias, los Comités de Bioética o el SAG cuestionan la necesidad de manipular (incluyendo extraer) ejemplares en áreas donde ya se han realizado estudios o se cuenta con especímenes en colecciones. Sin embargo, la literatura científica que registra la relevancia de colectar muestras adicionales en áreas previamente muestreadas es extensa y clara. Los siguientes tres estudios ilustran este punto. El primero tiene que ver con entender la evolución de niveles de contaminación ambiental en un área dada (DuBay \& Fuldner 2017), el segundo con entender la historia de enfermedades como el Síndrome Pulmonar por Hantavirus (Yates et al. 2002), y el tercero con cuantificar el efecto del cambio climático en la biota (Moritz et al. 2008). Además de mostrar claramente la utilidad de las colecciones científicas (ver el desarrollo de este argumento en Schindel \& Cook 2018), estos estudios tienen en común el haber sido posibles porque existen series de animales colectados en una misma área o localidad en distintas épocas. Lo anterior posibilitó el comparar la composición de ensambles de especies y los atributos de éstas a lo largo del tiempo. Este claro mensaje debe ser considerado por quienes promueven la reducción de la remoción de ejemplares en la naturaleza. 
Sugerencias a la regulación chilena de manipulación de vertebrados terrestres: D’ELíA, G. ET AL.

MANIPULACIÓN ANIMAL CON FINES CIENTÍFICOS Y DE CONSERVACIÓN La preocupación por el efecto que la manipulación de especímenes con fines científicos, en particular la remoción de los mismos, pueda tener sobre el estatus de las poblaciones naturales, si bien entendible, es injustificada. Los estudios científicos indican que la remoción con fines de investigación de individuos de poblaciones naturales sin problemas de conservación, no afecta el estatus de las mismas (e.g., Hope et al. 2018). Consecuentemente, la manipulación de individuos de vertebrados terrestres en poblaciones naturales debería estar regulada principalmente en términos del manejo de acuerdo a estándares que aseguren el bienestar animal (i.e., Refinamiento; ver Russell y Burch 1959), pero no en relación al tipo de manipulación, al número de animales que son manipulados, ni a la frecuencia temporal o espacial con que la manipulación se realiza.

De hecho, si lo que preocupa es el estatus de las poblaciones, otras actividades humanas deberían ser el centro de atención y no la manipulación científica. Por ejemplo, Escobar et al. (2015) muestran que más de la mitad $(52,4 \%)$ de los individuos del ratón de pelo largo Abrothrix hirta (referido como A. longipilis en la publicación) mueren en las plantaciones forestales del centro sur de Chile como consecuencia directa de las faenas forestales; los animales fueron aplastados por las máquinas involucradas en la cosecha o por los troncos al caer. La experiencia con solicitudes de colecta científica indica que los Comités de Bioética y el SAG no aprobarían una solicitud de remoción con fines científicos de la mitad de los ejemplares de una especie en un área dada.

De igual forma, otros factores afectan negativamente el estatus de las poblaciones naturales. Entre estos, junto a la destrucción de hábitat, los animales domésticos constituyen un problema mayor. Un análisis de la evidencia disponible estimó que en Estados Unidos los gatos matan anualmente entre 1,3 y 4 miles de millones de aves y entre 6,3 y 22,3 miles de millones de mamíferos (Loss et al. 2013). En la misma línea, un estudio en un poblado inglés mostró que en el transcurso de un año, 70 gatos domésticos cazaron un total de 1090 presas (535 mamíferos, 297 aves y 258 animales no identificados), estimándose que el 30\% de los gorriones que mueren en dicha localidad son cazados por gatos domésticos (Churcher y Lawton, 1987; ver resultados de estudios similares en van Heezik et al. 2010). No existen estudios de este tipo en Chile (pero véase SilvaRodríguez y Sieving 2011 y Muñoz-Zanzi et al. 2014 para una cuantificación relativa del efecto negativo de los gatos domésticos en la fauna), pero es razonable plantear que las tasas de depredación deberían ser similares. Aún cuando fuesen un orden de magnitud menor (i.e., 70 gatos cazando 53 mamíferos por año), si se considera el total de gatos presentes en zonas suburbanas y rurales del país, es fácil visualizar que los animales domésticos capturan muchos más ejemplares que los que son removidos de las poblaciones naturales en el transcurso de investigaciones científicas. Para contextualizar, la Colección de Mamíferos de la Universidad Austral de Chile, la mayor del país en su tipo e iniciada en 1974, tiene alrededor de 8000 especímenes catalogados (un ingreso promedio de ca. 178 individuos por año); un número mucho menor al de los individuos anualmente removidos de las poblaciones naturales por los gatos o como consecuencia de algunas faenas productivas. En este sentido, la siguiente afirmación de McNeely (2002:149) es oportuna: "It is ironic that governments who stringently police specimen collecting efforts in the name of conservation also grant extensive logging concessions and promote the replacement of natural forests by plantations, taking on an easy target while allowing the real culprits to flourish." (traducción: Es irónico que los gobiernos que en nombre de la conservación vigilan rigurosamente los esfuerzos de colecta de especímenes, también otorguen amplias concesiones de tala y promuevan el reemplazo de bosques naturales por plantaciones, poniendo dificultades a un blanco fácil mientras permiten que los verdaderos culpables prosperen).

Por lo tanto, es claro que la manipulación científica de animales, aún si la misma implicase siempre la remoción de ejemplares, no es la que pone en peligro a las poblaciones naturales. Los eventuales efectos que la misma puede tener en las poblaciones naturales deben ser entendidos como un costo mínimo, si es que lo tiene, asociado a la generación de conocimiento. Es más, se debe entender que una fracción importante de dicho conocimiento es relevante para diseñar estrategias más efectivas para la conservación de la biodiversidad (e.g., Thomson et al. 2018).

\section{RECOMENDACIONES \\ De esta forma, dada la crisis de la biodiversidad que actualmente enfrentamos, así como el genuino interés de la comunidad científica -inherente a la condición humana- de generar conocimiento, se plantean las siguientes recomendaciones en relación a las autorizaciones para su manipulación, como una forma de acelerar la generación de conocimiento sobre los vertebrados terrestres de Chile.}

Recomendación 1.- Que la injerencia de los Comités de Bioética se limite a temas de bienestar animal relacionadas al refinamiento (e.g., manipulación en terreno, condiciones de mantención de animales en bioterios, modalidades de eutanasia) y bioseguridad; es decir, que los Comités de Bioética no se involucren en relación al diseño de las investigaciones (e.g., tamaños muestrales, cobertura geográfica, tipo de muestra a tomar).

RECOMENDACión 2.- Que se avance hacia la implementación de un sistema de autorización de manipulación científica similar al vigente en Brasil, el país con mayor biodiversidad del planeta. En dicho sistema, existe una modalidad en 
que los investigadores cuentan con un permiso que los autoriza a colectar especímenes en el grupo taxonómico (e.g., roedores, lagartijas, anfibios) de su especialidad (demostrado por sus publicaciones) de forma permanente en todo el país, mientras dure su afiliación con una institución de investigación científica. El número de individuos a manipular queda bajo criterio del investigador. Una autorización especial se necesita para manipular ejemplares de especies con algún grado de amenaza o para trabajar en áreas protegidas. Ver detalles en http://www.icmbio.gov.br/ sisbio/

ReCOMENDACión 3.- Que sea obligatorio que los investigadores informen anualmente los detalles de sus capturas (e.g., especies, localidades) y que estos datos se integren a una base de datos pública y de acceso libre (e.g., nodo chileno del GBIF; no es el tema de este comentario por lo que solo se menciona que en relación al acceso a la información de la Biodiversidad, existe mucho espacio para mejorar en Chile). En caso de remoción de especímenes, una vez finalizadas las investigaciones, los mismos deberían ser depositados en colecciones científicas acreditadas (consignándose en informes y publicaciones los números de catálogo).

Cerramos este comentario esperando que marque el inicio de una discusión a nivel nacional en pos del establecimiento de una normativa de manipulación científica de animales en la naturaleza ajustada a la realidad biológica, y que no entorpezca la investigación científica.

\section{AGRADECIMIENTOS}

Lo expresado en este comentario nace de la experiencia de los autores y del intercambio de ideas con múltiples colegas y estudiantes. Nuestro agradecimiento a todos ellos. Luis Ebensperger, Juliana Vianna y un revisor anónimo proveyeron observaciones valiosas sobre una versión anterior de este comentario.

\section{REFERENCIAS}

Churcher, P.B., Lawton, J.H. 1987. Predation by domestic cats in an English village. Journal of Zoology 212(3):439-455.

D’Elía, G., Teta, P., Upham, N.S., Pardiñas, U.F.F., Patterson, B.D. 2015. Description of a new soft-haired mouse, genus Abrothrix (Sigmodontinae), from the temperate Valdivian rainforest. Journal of Mammalogy 96(4):839-853.

D’Elía, G., Hurtado, N., D’Anatro, A. 2016a. Alpha taxonomy of Dromiciops (Microbiotheriidae) with the description of 2 new species of monito del monte. Journal of Mammalogy 97(4):1136-1152.

D’Elía, G., Barria, R., Teta, P. 2016b. Primer registro del género
Notiomys Thomas 1890 (Rodentia, Cricetidae) para Chile. Therya 7: 315-319. DOI: 10.12933/therya-16-354.

Demangel, D., Sepúlveda, C., Jara, M., Pincheira-Donoso, D., NúÑEz, H. 2015. Liolaemus omorfi, a new lizard species from the Andes of northern Chile (Sauria, Liolaemidae). Boletín del Museo Nacional de Historia Natural, Chile, 64: 139-155.

DuBay, S.G., Fuldner, C.C. 2017. Bird specimens track 135 years of atmospheric black carbon and environmental policy. Proceedings of the National Academy of Sciences of the United States of America 114(43):11321-11326.

Escobar, M.A.H., Uribe, S.V., Chiappe, R., Estades, C.F. 2015. Effect of clearcutting operations on the survival rate of a small mammal. PLoS ONE 10(3): e0118883. https://doi. org/10.1371/journal.pone.0118883

Hope, A.G., Sandercock, B.K., Malaney, J.L. 2018. Collection of scientific specimens: benefits for Biodiversity Sciences and limited impacts on communities of small mammals. BioScience 68(1):35-42.

Loss, S.R., Will, T., MarRa, P.P. 2013. The impact of free-ranging domestic cats on wildlife of the United States. Nature Communications 4:1396. doi: 10.1038/ncomms2380.

MCNeEly, J.A. 2002. The role of taxonomy in conserving biodiversity. Journal for Natural Conservation 10(3):145153.

Moritz, C., Patton, J.L., Conroy, C.J., Parra, J.L., White, G.C., BeIssinger, S.R. 2008. Impact of a Century of Climate Change on Small-Mammal Communities in Yosemite National Park, USA. Science 322(5899):261-264.

Muñoz-Zanzi, C., Mason, M., Encina, C., Gonzalez, M., Berg, S. 2014. Household characteristics associated with rodent presence and Leptospira infection in rural and urban communities from Southern Chile. The American Journal of Tropical Medicine and Hygiene 90(3):497-506.

Ossa, G., Bonacic, C., Barquez, R.M. 2015. First record of Histiotus laephotis (Thomas, 1916) from Chile and new distributional information for Histiotus montanus (Phillipi and Landbeck, 1861) (Chiroptera, Vespertilionidae). Mammalia 79(4):457-461.

Ossa, G., Lilley, T.M., Ugarte-NúÑez, J., Ruokolainen, L., Vilches, K., Valladares-Faúndez, P., Yung, V. 2018. First record of Promops davisoni (Thomas, 1921) (Chiroptera, Molossidae) from Chile and a description of its echolocation calls. Mastozoología Neotropical, 25(1):129-137.

Palma, R.E., Rodríguez-Serrano, E. 2017. Systematics of Oligoryzomys (Rodentia, Cricetidae, Sigmodontinae) from southern Chilean Patagonia, with the description of a new species. Journal of Zoological Systematics and Evolutionary Research 56(2):280-299.

Russell, W.M.S., Burch, R.L. 1959. The Principles of Humane Experimental Technique, Methuen, London.

Schindel, D.E., Cook, J.A. 2018. The next generation of natural history collections. PloS Biology 16(7):e2006125. https:// doi.org/10.1371/journal.pbio.2006125

Silva-Rodríguez, E.A., Sieving, K.E. 2011. Influence of care of domestic carnivores on their predation on vertebrates. Conservation Biology 25(4):808-815.

Spotorno A.E., Zuleta, C., Walker, L., Manríquez, G., Valladares, P., Marín, J.C. 2013. A small, new gerbil 
Sugerencias a la regulación chilena de manipulación de vertebrados terrestres: D’ELíA, G. ET AL.

mouse Eligmodontia (Rodentia: Cricetidae) from dunes at the coasts and deserts of north-central Chile: molecular, chromosomic and morphological analyses. Zootaxa 3683(4):377-394.

Teta, P., D'Elía, G. 2016. Taxonomic notes on the long-clawed mole mice of the genus Geoxus (Cricetidae), with the description of a new species from an oceanic island of southern Chile. Hystrix, the Italian Journal of Mammalogy 27(2):1-10.

Troncoso-Palacios, J., Esquerré, D., Urra, F.A., Díaz, H.A., Castro-Pastene, C., Ruiz, M.S. 2018. The true identity of the New World iguanid lizard Liolaemus chillanensis Müller and Hellmich 1932 (Iguania: Liolaemidae) and description of a new species in the Liolaemus elongatus group. Zoological Studies 57(22): 1-19.

Thomson, S.A., Pyle, R.L., Ahyong, S., Alonso-Zarazaga, M., Ammirati, J., Araya, J.F., Ascher, J.S., Audisio, T.L., Azevedo-Santos, V.M., Bailly, N., Baker, W,J., Balke, M., Barclay, M.V.L., Barrett, R.L., Benine, R.C., Bickerstaff, J.R.M., Bouchard, P., Bour, R., Bourgoin, T., Boyko, C.B., Breure, A.SH., Brothers, D.J., Byng, J.W., Campbell, D., Ceríaco, L.M.P., Cernák, I., Cerretti, P., Chang, C.-H., Cho, S., Copus, J.M., Costello, M.J., Cseh, A., Csuzdi, C., Culham, A., D’Elía, G., D’Udekem D’Acoz, C., Daneliya, M.E., Dekker, R., Dickinson, E.C., Dickinson, T.A., van DiJK, P.P., DiJKstra, K.-DB., Dima, B., Dmitriev, D.A., Duistermaat, L., Dumbacher, J.P., Eiserhardt, W.L., Ekrem, T., Evenhuis, N.L., Faille, A., Fernández-Triana, J.L., Fiesler, E., Fishbein, M., Fordham, B.G., Freitas, A.V.L., Friol, N.R., Fritz, U., Frøslev, T., Funk, V.A., Gaimari, S.D., Garbino, G.S.T., Garraffoni, A.R.S., Gem,l J., Gill, A.C., Gray, A., Grazziotin, F.G., Greenslade, P., Gutiérrez, E.E., Harvey, M.S., Hazevoet, C.J., He, K., He, X., Helfer, S., Helgen, K.M., van Heteren, A.H., Hita Garcia, F., Holstein, N., Horváth, M.K., Hovenkamp, P.H., Hwang, W.S., Hyvönen, J., Islam, M.B., Iverson, J.B., Ivie, M.A., JAAFAR, Z., JACKSON, M.D., JAYAT, J.P., JohnSON, N.F., Kaiser, H., Klitgård, B.B., Knapp, D.G., Kojima, J., Kõlualg, U., Kontschán, J., Krell, F.-T., KrisaiGreilhuber, I., Kullander, S., Latella, L., LattKe, J.E., Lencioni, V., Lewis, G.P., Lhano, M.G., Lujan, N.K., Luksenburg, J.A., Mariaux, J., Marinho-Filho,
J., Marshall, C.J., Mate, J.F., McDonough, M.M., Michel, E., Miranda, V.F.O., Mitroiu, M.-D., Molinari, J., Monks, S., Moore, A.J., Moratelli, R., Murányi, D., Nakano, T., Nikolaeva, S., Noyes, J., Ohl, M., Oleas, N.H., Orrell, T., Páll-Gergely, B., Pape, T., Papp, V., Parenti, L.R., Patterson, D., Pavlinov, I.Y., Pine, R.H., Poczai, P., Prado, J., Prathapan, D., Rabeler, R.K., Randall, J.E., Rheindt, F.E., Rhodin, A.G.J., Rodríguez, S.M., Rogers, D.C., Roque. Fd.O., Rowe, K.C., Ruedas, L.A., Salazar-Bravo, J., Salvador, R.B., Sangster, G., Sarmiento, C.E., Schigel, D.S., Schmidt, S., Schueler, F.W., Segers, H., Snow, N., Souza-Dias, P.G.B., Stals, R., Stenroos, S., Stone, R.D., Sturm, C.F., Štys, P., Teta, P., Thomas, D.C., Timm, R.M., Tindall, B.J., Todd, J.A., Triebel, D., Valdecasas, A.G., Vizzini, A., Vorontsova, M.S., de Vos, J.M., Wagner, P., Watling, L., Weakley, A., Welter-Schultes, F., Whitmore, D., Wilding, N., Will, K., Williams, J., Wilson, K., Winston, J.E., Wüster, W., Yanega, D., Yeates, D.K., Zaher, H., Zhang, G., Zhang, Z.-Q., Zhou, H.-Z. 2018. Taxonomy based on science is necessary for global conservation. PLoS Biology 16(3):e2005075. https://doi.org/10.1371/journal. pbio. 2005075

Valladares, P., Álvarez, N., Urrutia, N., Nassar, C., Iriarte, A. 2015. Primer registro de Abrothrix jelskii (Thomas 1824) para Chile. Gayana 79(2):223-225.

van Heezik, Y., Smyth, A., Adams, A., Gordon, J. 2010. Do domestic cats impose an unsustainable harvest on urban bird populations? Biological Conservation 143(1):121130.

Yates, T.L., Mills, J.M., Parmenter, C.A., Ksiazek, T.G., Parmenter, R.R., Vande Castle, J.R., Calisher, C.H., Nichol, S.T., Аbbott, K.D., Young, J.C., Morrison, M.L., Beaty, B.J., Dunnum, J.L., Baker, R.J., Salazar-Bravo, J. Peters, C.J. 2002. The Ecology and Evolutionary History of an Emergent Disease: Hantavirus Pulmonary Syndrome: Evidence from two El Niño episodes in the American Southwest suggests that El Niño-driven precipitation, the initial catalyst of a trophic cascade that results in a delayed density-dependent rodent response, is sufficient to predict heightened risk for human contraction of hantavirus pulmonary syndrome. BioScience 52(11):989-998.

Recibido: 23.01.2019

Aceptado: 04.04.2019 